\title{
Role of CT scan in paranasal sinus pathology
}

\author{
Mohit Srivastava $^{1 *}$, Himani Singh ${ }^{2}$, Waseem Ahmed ${ }^{3}$, Sachin Agarwal $^{4}$ \\ ${ }^{1}$ Associate Professor, ${ }^{2,4}$ PG Resident, ${ }^{3}$ Senior Resident, Dept. of ENT, Saraswathi Institute of Medical Sciences, Hapur, Uttar Pradesh, \\ India
}

\section{*Corresponding Author: Mohit Srivastava}

Email: dr.mohit141180@gmail.com

\begin{abstract}
CT Scans use a narrow X-Ray beam that circles around the part of body to be scanned. The data is transmitted to a computer, which build up 3D cross sectional picture. Although CT Scans cannot distinguish between inflammation and infection, they correlate with extent of disease. CT Scans have been very useful in assessing severity of the disease or response to treatment in chronic Rhinosinusitis. It is also the most reliable radiological examination to identify a fungus ball. The latest CT machines allow a complete set of axial slices to be made within a few seconds. The CT scan proved to be an excellent imaging tool as it can accurately diagnose \& differentiate benign $\&$ malignant lesions, can describe the masses in terms of their origin, nature, extension, \& involvement. Computerized tomography (CT) is considered the gold standard for preoperative evaluation of PNS diseases for appropriate patient selection for functional endoscopic sinus surgery (FESS). CT has some medico-legal importance as well. This is a prospective study conducted on 30 patients (from September 2018 to May 2019). Both male and female patients of different age groups with clinically suspected PNS diseases were enrolled for the study. Both axial and coronal images were acquired using multi-detector CT unit. The paranal sinuses were evaluated for: mucosal thickening, wall of sinus, fluid level, soft tissue extension, hyperdensities and masses, blockage of osteomeatal complex, and pattern of bony involvement. There was a male predominance of $54 \%$ as compared to females $46 \%$. The chief presenting complaint was of nasal obstruction (49\%), followed by a headache $(40.8 \%)$, nasal discharge $(30.5 \%)$, facial pain $(20 \%)$, and others $(37.7 \%)$. The most common CT diagnosis was chronic sinusitis (13/30) followed by polyp (7/30) and fungal sinusitis (5/30). In this study, maxillary sinus (83.5\%) was the most commonly involved sinus followed by ethmoid sinus (74.7\%), sphenoid sinus (43.9\%), and frontal sinus (54.9\%). CT was able to characterize the PNS diseases along with their extension. It could also delineate the bony involvement of PNS diseases. CT may be used as gold standard imaging modality for evaluating the Paranasal Sinus diseases.
\end{abstract}

Keywords: Paranasal sinus pathology, Chronic sinusitis, Headache, Computed tomography.

\section{Introduction}

Pathological lesions of the paranasal sinuses include a wide spectrum of conditions ranging from inflammation to neoplasms both benign and malignant. Most patient of common cold present with symptoms of nasal discharge, nasal obstruction, headache, h/o allergy etc. The patient of paranasal mass usually present with facial deformity, swelling or repeated episodes of epistaxis. Preliminary investigation by standard sinus radiographs are sub optimal in displaying regional morphology of Osteomeatal area, Ethmoid\& Sphenoid sinuses and anatomical variants. Further there is overlapping between these structures hence these are insufficient to diagnose and as a guide to endoscopic sinus surgery \& modified PNS surgeries

The CT scan proved to be an excellent imaging tool as it can accurately diagnose \& differentiate benign \& malignant lesions, can describe the masses in terms of their origin, nature, extension, \& involvement. Now with the unique ability of CT to image both the bones and soft tissues, direct coronal scanning and sagittal reconstruction imaging the space occupying lesions. By acting as a Roadmap preoperative CT scan PNS has proved to reduce the post operative complications of FESS \& other surgeries.

\section{Objectives}

1. To evaluate the CT findings in patients with paranasal sinus pathologies and correlate it with clinical findings.

2. To assess the reliability of CT PNS for early diagnosis of PNS pathology.
3. To assess the severity and extent of inflammatory and malignant pathologies of PNS.

4. To differentiate benign from malignant lesions.

Computerized tomography (CT) is considered the gold standard for preoperative evaluation of PNS diseases for appropriate patient selection for functional endoscopic sinus surgery (FESS). CT has some medico-legal importance as well. ${ }^{1,2}$

Coronal imaging plane offers the best visualization of the drainage pathways of the sinuses, whereas some drainage pathways (such as sphenoid sinus ostia) and sinus walls, oriented close to the coronal plane, are better seen on axial images. ${ }^{3,4}$

Contrast enhanced CT scans are obtained only in patients who are acutely ill and suspected of having a complication of acute sinusitis. ${ }^{5}$

\section{Materials and Methods}

This was a prospective study conducted on 30 patients (from September 2018 to May 2019) after the approval of the Medical Research and Ethical Committee of the Institution. Both male and female patients of different age groups with clinically suspected PNS diseases were enrolled for the study. All postoperative cases and patients with any traumatic conditions involving PNS were excluded from the study. The detailed clinical assessment was performed, and their informed consent was obtained before subjecting them for the imaging modality. 
A detailed clinical history including age, sex, occupation, duration of symptoms and specific complaints such as headache, nasal obstruction, nasal discharge, and facial pain were taken. Family history and personal history were also recorded. A thorough clinical examination was done, and obtained data were recorded, and a provisional diagnosis was prepared. Both axial and coronal images were acquired using multi-detector $\mathrm{CT}$ unit.

The sinuses were evaluated for the following parameters: mucosal thickening, wall of sinus, fluid level, soft tissue extension, hyperdensities and masses, blockage of osteomeatal complex, and pattern of bony involvement. The results were recorded and expressed as sinus distribution, side of involvement, frequency of deviated nasal septum (DNS), concha bullosa, frequency of ostemeatal unit involvement, and provisional CT diagnosis.

\section{Result}

A total of 30 patients fulfilling the inclusion criteria, were included in the present study. A descriptive comparative analysis of imaging findings was conducted, tabulated, and results were derived.

The majority of the cases were in the age group of $16-$ 30 years $(33 \%)$ and least number of patients were in age group of $71-80$ years $(0.76 \%)$. The youngest patient was 12 year old, and eldest patient was 75 year old. There was a male predominance of $54 \%$ as compared to females $46 \%$. The male to female ratio was $1.17: 1$. The chief presenting complaint was of nasal obstruction (49\%), followed by a headache $(40.8 \%)$, nasal discharge $(30.5 \%)$, facial pain $(20 \%)$, and others $(37.7 \%)$.

The most common CT diagnosis was chronic sinusitis (13/30) followed by polyp (7/30) and fungal sinusitis (5/30).

In this study, maxillary sinus $(83.5 \%)$ was the most commonly involved sinus followed by ethmoid sinus (74.7\%), sphenoid sinus (43.9\%), and frontal sinus (54.9\%). DNS was noted toward right side in $43.9 \%$ cases and toward left side in $28.5 \%$ of cases. Concha bullosa was noted in $(30 \%)$ cases of which left sided involvement was noted in $(13.05 \%)$, right siuded in $(10.8 \%)$ and bilateral in 8 caess (6.92\%) [Fig. 1],[Fig. 2]

Ostiomeatal unit (OMU) obstruction was noted in 49 cases $(53.8 \%$ ), of which left sided was noted in $26.3 \%$, right sided in $24.1 \%$, and bilateral involvement was seen in $3.2 \%$ cases. The most common pattern of involvement was sinonasal polyposis $(41.7 \%)$, followed by osteomeatal $(28.5 \%)$, infundibular $(15.3 \%)$, sphenoethmoidal recess (5.4\%), and sporadic pattern (2.1\%) [Fig. 3], [Fig. 4], [Fig. 5], [Fig. 6], [Fig. 7].

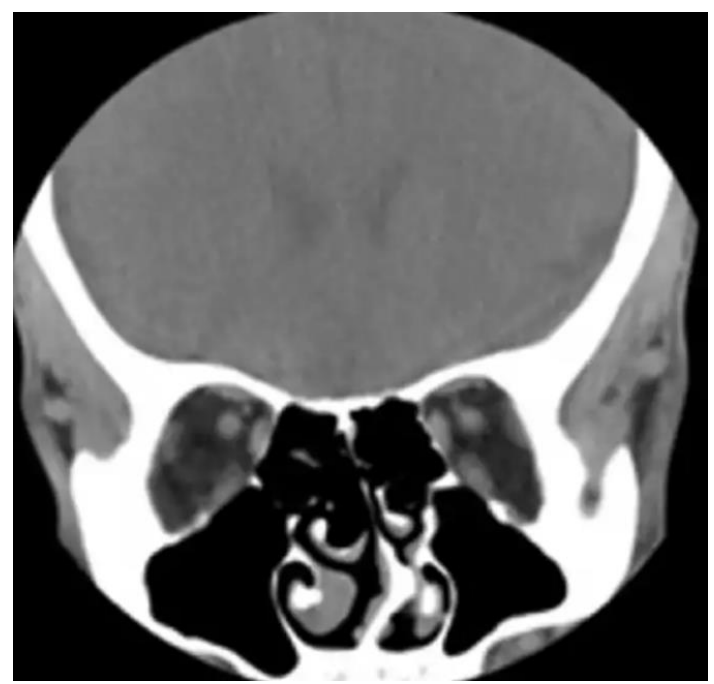

Fig. 1

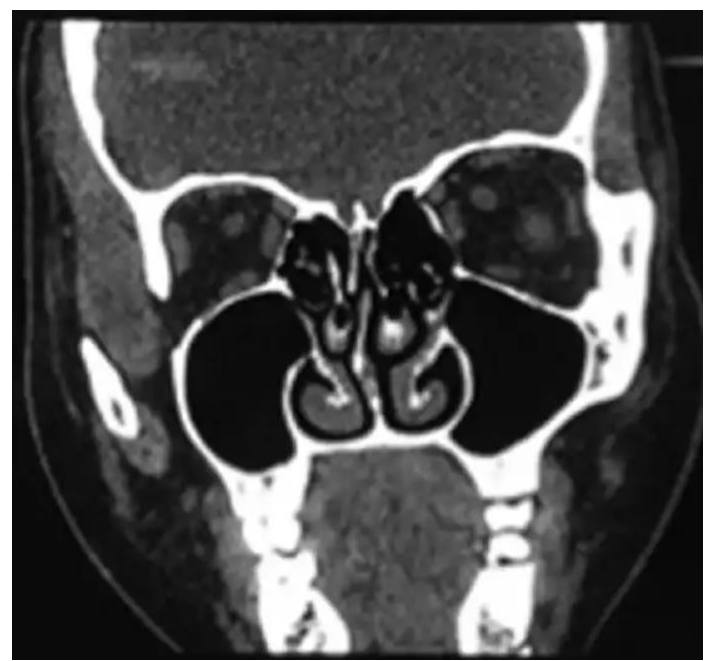

Fig. 2

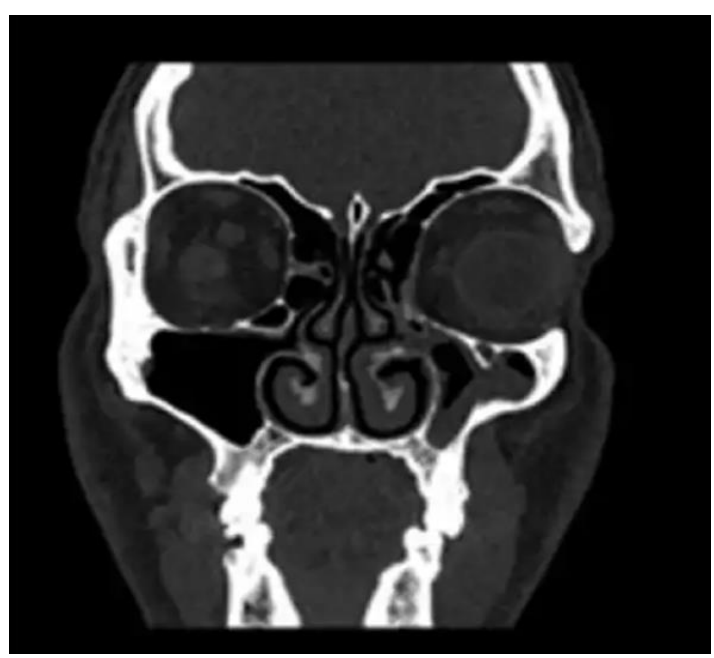

Fig. 3 


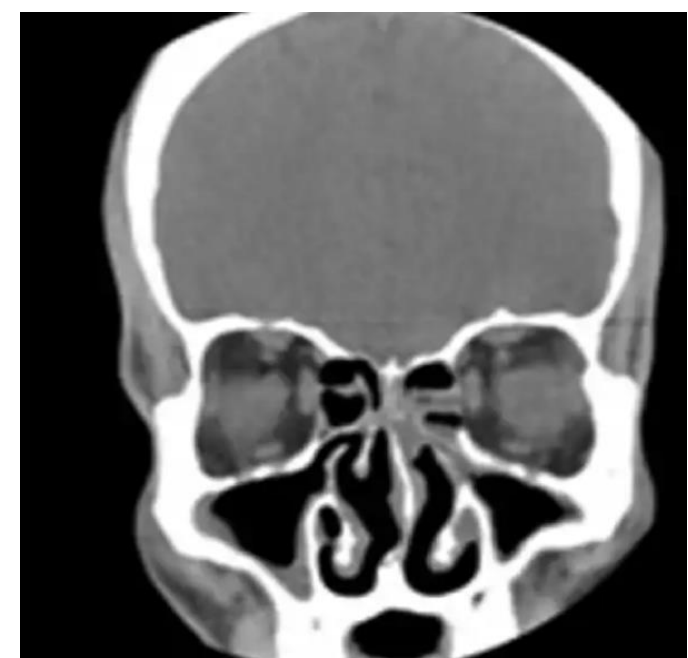

Fig. 4

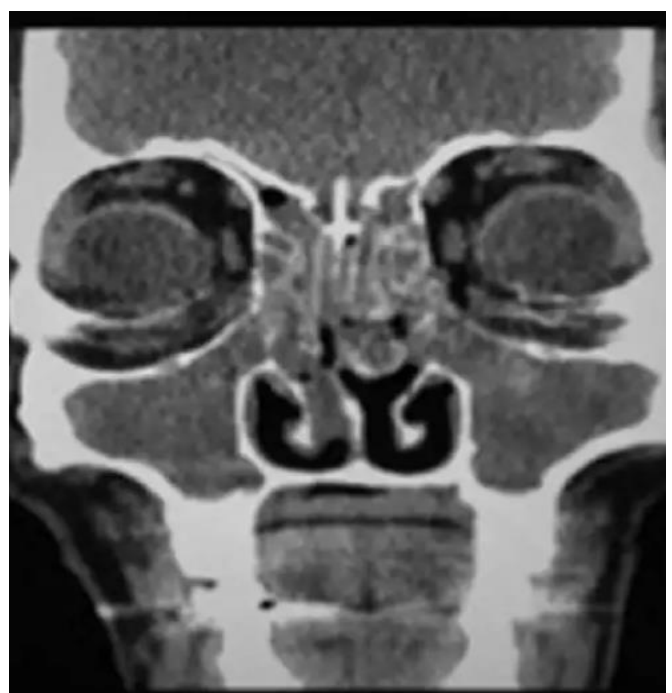

Fig. 5

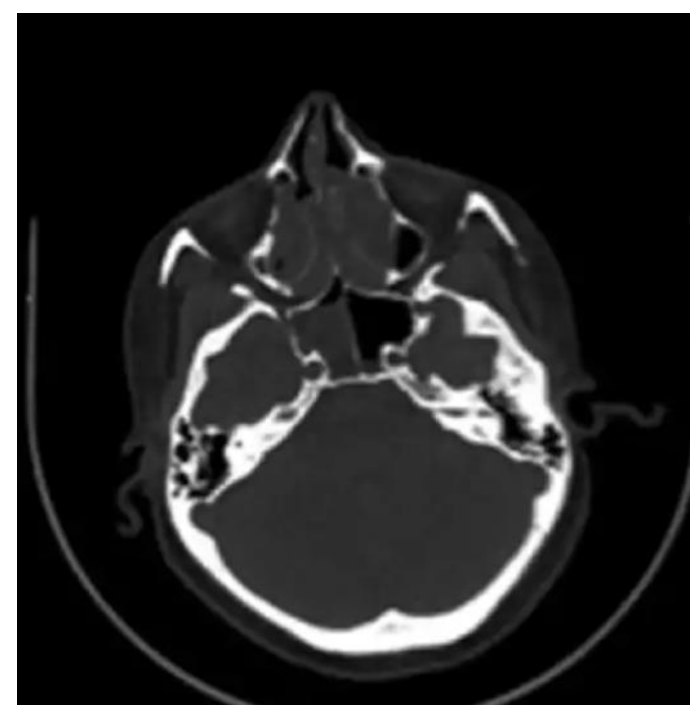

Fig. 6

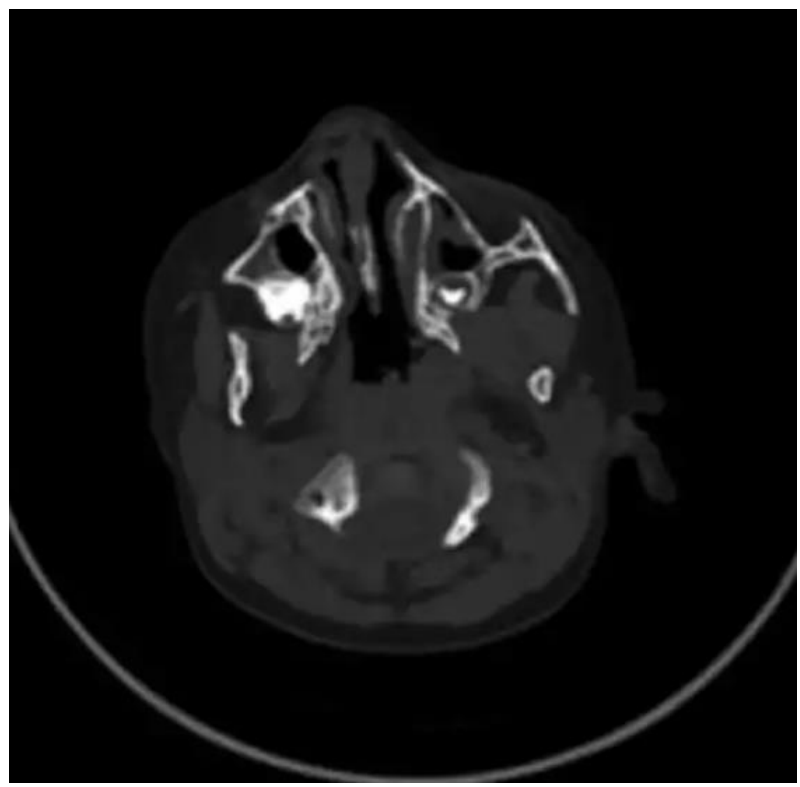

Fig. 7

Other common anatomical variants encountered in our study were paradoxical middle turbinate, bulla ethmoidalis, and Haller cells.

In the present study CT, detected bone involvement, i.e., erosion or destruction of sinus walls in six cases $(4.6 \%)$ while clinically, no bony involvement was detected in these cases. In the present study, CT reported 16 cases as fungal sinusitis, out of which only 6 cases were confirmed by histopathology

\section{Conclusion}

CT was able to characterize the PNS diseases along with their extension. It could also delineate the bony involvement of PNS diseases. Preoperative CT enabled the surgeon to visualize the drainage pathways, anatomical and critical variants in PNSs thus allowing effective management of the patient. However, a potential pitfall was its inability to accurately differentiate in cases of fungal sinusitis and highdensity secretions. CT may be used as gold standard imaging modality for evaluating the PNS diseases.

\section{Financial Support and Sponsorship: Nil.}

\section{Conflicts of Interest: None.}

\section{References}

1. Lund VJ, Savy L, Lloyd G. Imaging for endoscopic sinus surgery in adults. J Laryngol Otol 2000;114:395-7.

2. Chopra H. Nasal polyps: A clinical, histopathological and radiological profile. Indian J Otolaryngol Head Neck Surg 2008;60:112-6.

3. Duvoisin B, Landry M, Chapuis L, Krayenbuhl M, Schnyder $\mathrm{P}$. Low-dose CT and inflammatory disease of the paranasal sinuses. Neuroradiol 1991;33:403-6.

4. Hoang JK, Eastwood JD, Tebbit CL, Glastonbury CM. Multiplanar sinus CT: A systematic approach to imaging before functional endoscopic sinus surgery. AJR Am J Roentgenol 2010;194:W527-36. 
5. Fatterpekar GM, Delman BN, Som PM. Imaging the paranasal sinuses: Where we are and where we are going. Anat Rec (Hoboken) 2008;291:1564-72.

How to cite this article: Srivastava M, Singh $\mathrm{H}$, Ahmed W, Agarwal S. Role of CT scan in paranasal sinus pathology. Indian $J$ Anat Surg Head Neck Brain 2019;5(2):51-4. 